\title{
STRONG UNIQUENESS FOR SECOND ORDER ELLIPTIC OPERATORS WITH GEVREY COEFFICIENTS
}

\author{
Ferruccio Colombini, Cataldo Grammatico, Daniel Tataru
}

\begin{abstract}
We consider here the problem of strong unique continuation property at zero, for second order elliptic operators $P=P(x, D)$ with complex coefficients. For such operators we obtain this property by means of suitable Carleman's estimates in Gevrey classes of appropriate index. This index depends on the spread of the cone image of the principal symbol $p$ of $P$ evaluated at zero, that is $p\left(0, \mathbf{R}^{N} \backslash\{0\}\right)$. Secondly by using similar techniques we deal with the strong unique continuation property in suitable Gevrey classes for some fourth order elliptic operators with real coefficients.
\end{abstract}

\section{Introduction}

We consider here the problem of strong unique continuation property for second order elliptic operators with complex coefficients. Throughout the paper $\Omega$ is an open neighborhood of the origin in $\mathbf{R}^{N}$. Let $C_{b}^{\infty}(\Omega)$ denote the space of functions in $C^{\infty}(\Omega)$ which are flat at the origin.

For a partial differential operator $P$ with smooth coefficients in $\Omega$ we shall adopt the following

Definition 1.1. We say that $P$ has the strong unique continuation property at 0 if whatever $P u=0$ in $\Omega$ and $u \in C_{b}^{\infty}(\Omega)$ then $u \equiv 0$ in a neighborhood of zero.

Let now $P$ be a second order, elliptic operator with smooth coefficients defined in an open neighborhood of the origin in $\mathbf{R}^{2}$. We denote by $P_{2}$ the principal part of $P$. If $P_{2}$ has simple complex characteristics then, as a consequence of the results in [1] and [2], we have the following:

1. if $P_{2}\left(0, D_{x}\right)$ is real, then $P$ has the strong unique continuation property at 0 .

2. if $P_{2}\left(0, D_{x}\right)$ is not real, then there exists a $C^{\infty}$ function $a$ flat at zero (more exactly in a suitable Gevrey class) and a function $u \in C^{\infty}$ flat at zero, not identically zero, such that

$$
P u+a u=0 .
$$

Received by the editors January 31, 2005.

2000 Mathematics Subject Classification. Primary 35B60; Secondary 35J15, 35J30. 
In these notes we study the case in which $P$ has Gevrey coefficients. In 1981 Lerner [16] considered the two dimensional case and proved that if $P$ is a second order elliptic operator with simple characteristics and with Gevrey coefficients of order $s$ then $P$ has the strong unique continuation property at zero if the Gevrey index $s$ is smaller than a quantity depending on the cone $p_{2}\left(0, \mathbf{R}^{2}\right)$.

Lerner's results in $\mathbf{R}^{2}$ (see [16]) are presented in the Appendix. In this paper we extend his results to $\mathbf{R}^{N}$. The strong uniqueness at 0 for $P$ is obtained provided that $P$ has Gevrey coefficients of order smaller than a quantity depending only of the spread of the image cone $P_{2}\left(0, \mathbf{R}^{N}\right)$. (see Theorem 2.2 below).

Our strategy for the proof is as follows. Using linear transformations we reduce the problem to the case when $P_{2}(0, D)=\Delta+i Q$, with $Q=\sum \lambda_{k} \partial_{x_{k}}^{2}$. We note that the spread of the cone $P_{2}\left(0, \mathbf{R}^{N}\right)$ depends only on the smallest and the largest of the numbers $\lambda_{k}$. Then we proceed as in [22] to prove a Carleman estimate for the operator $P$ with a suitable weight which is singular at 0 . The required strength of the singularity of the weight at 0 is suggested by Lemma 3.2 .

The same method leads to a strong uniqueness result for the product of two elliptic operators $P Q$ with smooth, real coefficients. This improves an earlier result in [8]. Without any restriction in generality we take $P(0, D)=\Delta$. Obviously if $Q$ is also proportional to Laplace operator then much stronger results hold. In this case, many authors (see e.g. [2],[4],[9],[11],[12],[18],[19],[21]) have studied the same type of problem for $u \in C_{b}^{\infty}(\Omega)$ satisfying

$$
\left|\Delta^{h} u(x)\right| \leq\left|W_{0}(x)\right||u(x)|+\left|W_{1}(x)\right||\nabla u(x)|+\cdots+\left|W_{h}(x)\right|\left|\nabla^{h} u(x)\right|
$$

where $h \in \mathbf{N}$. In [5] it was proved that the strong unique continuation holds for the inequality (2) with $\left|W_{l}(x)\right| \leq \mathcal{C}_{l}|x|^{l-2 h}, \quad l=0, \ldots, h$ with small $\mathcal{C}_{h}$.

With a slight abuse of notations we say that the relation (2) has the strong unique continuation property if the only function $u(x) \in C_{\mathrm{b}}^{\infty}(\Omega)$ satisfying (2) is the zero function.

For general elliptic equalities and inequalities as in (2) counterexamples are given in [1], [3], [7], [13], [17], [23].

For the sake of completeness we note that the study of the strong unique continuation property for solutions of differential equalities and inequalities of second order elliptic operators with smooth and non-smooth real coefficients has a much longer history and is essentially complete. For more details we refer the reader to [12], [13], [15].

\section{Results}

We begin with a preliminary observation,

Lemma 2.1. ([20]) If $P(D)$ be a second order, elliptic operator in $\mathbf{R}^{N}$ and $p$ is its principal symbol then $p\left(\mathbf{R}^{\mathrm{N}} \backslash\{0\}\right)$ is either a convex cone of $\mathbf{C} \backslash\{0\}$, or it is $\mathbf{C} \backslash\{0\}$. The second alternative can only hold in dimension $N=2$. 
Now we can state our main result:

Theorem 2.2. Let $P$ be a second order elliptic operator with Gevrey coefficients of order $s>1$ which is defined in an open neighborhood of the origin in $\mathbf{R}^{\mathrm{N}}$. Let $p$ be its principal symbol.

If $p\left(0, \mathbf{R}^{\mathrm{N}} \backslash\{0\}\right)$ is a convex cone with angle $2 \phi, 0<\phi<\frac{\pi}{2}$ and if

$$
s<1+\frac{1-\sin \phi}{2 \sin \phi}
$$

then $P$ has the strong unique continuation property at zero.

One may ask whether the index obtained in (3) is optimal. We do not know the answer to this. So far we only know of $C^{\infty}$ counterexamples for this problem, see Alinhac's [1]. Whether Alinhac's ideas can be extended to the Gevrey class remains an open question.

Using a similar approach we obtain the following:

Theorem 2.3. Let $P(x, D)$ be a fourth order, elliptic operator with Gevrey coefficients of order $s>1$, defined in an open neighborhood $V$ of the origin in $\mathbf{R}^{N}$. Suppose that

$$
P(x, D)=L(x, D) Q(x, D)+a(x)
$$

where $L$ and $Q$ are second order elliptic partial differential operators.

Assume that the principal parts $L_{2}, Q_{2}$ of $L$ and $Q$ satisfy

$$
L_{2}(0, D)=\Delta, \quad Q_{2}(0, D)=\mu_{1}^{2} \partial_{x_{1}}^{2}+\cdots+\mu_{N}^{2} \partial_{x_{N}}^{2}, \quad \mu_{i}>0
$$

Then $P$ has the strong unique continuation property at 0 provided that

$$
s<1+\frac{\min _{i} \mu_{i}}{\max _{i, j}\left|\mu_{i}-\mu_{j}\right|} .
$$

For $N=2$ this result was proved in [6]. For larger $N$ it improves the result in [8] where the strong unique continuation property was proved only if $s<$ $1+\frac{1}{2} \frac{\min _{i} \mu_{i}}{\max _{i, j}\left|\mu_{i}-\mu_{j}\right|}$.

Remark 2.1. We note that the above operators are Gevrey hypoelliptic because their coefficients belong to Gevrey class of order s (denoted by $G^{s}$ ), hence Pu=0 implies $u \in G^{s}$. 
2.1. Proofs. Proof of Theorem 2.2 - We first argue that by making a rotation $P \rightarrow e^{i \theta} P$ we can insure that $\Re p(0, \xi)>0$. Indeed, let

$$
c=\max _{\theta} \min _{|\xi|=1} \Re\left(e^{i \theta} p(0, \xi)\right) \text {. }
$$

By Lemma 2.1 we know that $c \geq 0$. On the other hand, if we had $c=0$ then at the min-max point we should have

$$
\Re\left(e^{i \theta} p(0, \xi)\right)=0 \quad, \quad \partial_{\xi, \theta} \Re\left(e^{i \theta} p(0, \xi)\right)=0 .
$$

But this implies that $p(0, \xi)=0$, contradicting the ellipticity condition.

If $\Re p(0, \xi)>0$ then we can diagonalize it by means of an orthogonal transformation. Rescaling along the principal directions $x_{k} \rightarrow c_{k} x_{k}$ we can insure that $\Re p(0, \xi)=|\xi|^{2}$. Then we use another orthogonal transformation to diagonalize $\Im p(0, \xi)$. Thus we may take $p(0, \xi)$ of the form

$$
p(0, \xi)=|\xi|^{2}+i \sum \lambda_{k} \xi_{k}^{2}, \quad \lambda_{k} \in \mathbf{R}
$$

After another rotation in $\mathbf{C}$ and a rescaling along the principal directions we can also insure that

$$
\max _{k} \lambda_{k}=-\min _{k} \lambda_{k}=\lambda>0 .
$$

All these transformations preserve the spread of the cone $p\left(0, \mathbf{R}^{N} \backslash\{0\}\right)$.

The angle of the cone is given by

$$
\sin \phi=\frac{\lambda}{\sqrt{1+\lambda^{2}}} .
$$

We also note that after the reduction the cone lies on the right semiplane and it is symmetric with respect to the positive real axis.

We now prove Carleman estimates for the operator $P$ with respect to the singular weight

$$
\varphi(x)=r^{-\alpha}, \quad r=|x|
$$

with

$$
\frac{1}{s-1}>\alpha>\frac{2 \sin \phi}{1-\sin \phi}
$$

The Carleman estimates have the form

$$
\tau^{-1}\left\||r \nabla \varphi|^{-\frac{1}{2}} e^{\tau \varphi} v\right\|_{2, \tau}^{2} \leq c\left\|e^{\tau \varphi} P(x, D) v\right\|_{L^{2}}^{2} \quad \tau>\tau_{0},
$$

where $v$ is any smooth function with support in the punctured disk $0<|x|<\varepsilon$ for some $\varepsilon>0$ fixed. Moreover the constant $c$ in (8) can be taken independent of the support of the function $v$.

The norm on the left hand side is defined by

$$
\|v\|_{2, \tau}^{2}=\left\|\partial^{2} v\right\|_{L^{2}}^{2}+\left\|\tau^{2}|\nabla \varphi|^{2} v\right\|_{L^{2}}^{2} .
$$

Given the Carleman estimate (8) the strong uniqueness follows in a standard manner. 
In fact, let $\chi$ be a smooth function supported in $\{|x|<\varepsilon\}$ and equal to 1 in $\{|x|<\varepsilon / 2\}$ and let us put, for $j \in \mathbf{N}, \psi_{j}(x)=\psi(j x)$, where $\psi \in C^{\infty}\left(\mathbf{R}^{N}\right)$, radial increasing in $|x|$, is such that

$$
\psi(x)=\left\{\begin{array}{lll}
0 & \text { if } & |x| \leq 1 / 2 \\
1 & \text { if } \quad|x| \geq 1
\end{array}\right.
$$

Let now be $u$ any smooth function flat at zero such that $P u=0$, then the functions $\psi_{j} \chi u$ satisfy the estimate (8) with a constant $c$ independent of $j$. Taking into account Remark 2.1 and Lemma 3.2, we know that $u$ and its derivatives must decay at 0 faster that $e^{-\tau \varphi}$ for all $\tau>0$. Thus passing to the limit the same inequality (8) is satisfied by $\chi u$.

Moreover

$$
P(\chi u)=[P, \chi] u,
$$

where the commutator is supported in $\{\varepsilon / 2<|x|<\varepsilon\}$. Then applying (8) to $\chi u$ we obtain

$$
\tau^{-1}\left\||r \nabla \varphi|^{-\frac{1}{2}} e^{\tau \varphi} \chi u\right\|_{2, \tau}^{2} \leq c e^{\tau \varphi(\varepsilon / 2)}\|[P(x, D), \chi] u\|_{L^{2}}^{2} \quad \tau>\tau_{0} .
$$

Letting $\tau \rightarrow \infty$ this shows that $u=0$ in $\{|x|<\varepsilon / 2\}$.

The Carleman estimates (8) follow in turn from the strong pseudoconvexity condition for the function $\varphi$ with respect to the operator $P(x, D)$. We define the conjugated operator

$$
P_{\tau}(x, D)=e^{\tau \varphi} P(x, D) e^{-\tau \varphi}=P(x, D+i \tau \nabla \varphi),
$$

whose $\tau$-depending symbol $(x \neq 0)$ is

$$
p_{\tau}(x, \xi)=p(x, \xi+i \tau \nabla \varphi) .
$$

The strong pseudo-convexity condition in our case has the form

$$
\left\{\Re p_{\tau}, \Im p_{\tau}\right\}>\frac{c}{r}(|\xi|+\tau|\nabla \varphi|)^{3} \quad \text { on } \quad \operatorname{char} p_{\tau} .
$$

In order to prove that the Carleman estimates (8) are a consequence of the strong pseudo-convexity (10) we choose to rely on classical results in [10]. However, some additional care is required since the weight $\varphi$ is singular at 0 . We outline the main steps and leave the details to the reader.

Step 1. We consider a dyadic decomposition for a punctured neighborhood $V$ of the origin,

$$
V \subseteq \bigcup_{k<k_{0}} A_{k}
$$

where the $A_{k}$ 's are overlapping dyadic annuli

$$
A_{k}=\left\{2^{k-1}<|x|<2^{k+1}\right\} .
$$

Correspondingly we take a smooth partition of unity

$$
1=\sum \chi_{k}(x), \quad \text { supp } \chi_{k} \subseteq A_{k} .
$$


Step 2. We show that (8) holds for $u$ supported within a single dyadic region $A_{k}$. For this we rescale $A_{k}$ into a unit annulus by setting $y=2^{-k} x$. After rescaling (8) becomes

$$
\sigma^{-1}\left\|e^{\sigma \varphi} v\right\|_{2, \sigma}^{2} \leq c\left\|e^{\sigma \varphi} P\left(2^{k} y, D_{y}\right) v\right\|_{L^{2}}^{2}, \quad \sigma=\tau 2^{-\alpha k}, \quad y \in A_{1} .
$$

At the same time, the pseudoconvexity condition (10) rescales into a similar condition but with $\tau$ replaced by $\sigma$. In this context, (11) is a direct consequence of the results in [10].

Step 3. We assemble the localized results using the above partition of unity.

Precisely, applying (8) to $\chi_{k} v$ we obtain

$$
\tau^{-1}\left\|e^{\tau \varphi}|r \nabla \varphi|^{-\frac{1}{2}} \chi_{k} v\right\|_{2, \tau}^{2} \leq c\left(\left\|e^{\tau \varphi} \chi_{k} P(x, D) v\right\|_{L^{2}}^{2}+\left\|e^{\tau \varphi}\left[\chi_{k}, P(x, D)\right] v\right\|_{L^{2}}^{2}\right) .
$$

We sum with respect to $k$ to obtain

$$
\tau^{-1}\left\|e^{\tau \varphi}|r \nabla \varphi|^{-\frac{1}{2}} v\right\|_{2, \tau}^{2} \leq c\left(\left\|e^{\tau \varphi} P(x, D) v\right\|_{L^{2}}^{2}+\sum_{k}\left\|e^{\tau \varphi}\left[\chi_{k}, P(x, D)\right] v\right\|_{L^{2}}^{2}\right) .
$$

Finally, a direct computation shows that the commutator terms are negligible compared to the left hand side. Hence (8) follows.

It remains to prove the pseudoconvexity condition (10). We begin with several simplifications. The first is to observe that without any restriction in generality we can freeze the coefficients of $P$ at 0 . This is because for small $|x|$ the effect of the coefficients is negligible in the above inequality. Secondly, we note that by homogeneity considerations we can take $\tau=1$. Thus if we set

$$
p_{\varphi}(x, \xi)=p(0, \xi+i \nabla \varphi), \quad x \neq 0,
$$

we need to prove that

$$
\left\{\Re p_{\varphi}, \Im p_{\varphi}\right\}>\frac{c}{r}(|\xi|+|\nabla \varphi|)^{3} \text { on } \operatorname{char} p_{\varphi},
$$

where

$$
\operatorname{char} p_{\varphi}=\left\{\Re p_{\varphi}=\Im p_{\varphi}=0\right\} .
$$

Taking into account the remark made after estimate (8) we need to establish inequality (13) out of the origin.

Since

$$
\nabla \varphi=-\alpha x r^{-\alpha-2}
$$

we get

$$
\Re p_{\varphi}=\sum\left(\xi_{i}^{2}-\alpha^{2} x_{i}^{2} r^{-2 \alpha-4}\right)+2 \alpha \sum \lambda_{i} x_{i} \xi_{i} r^{-\alpha-2}
$$

and

$$
\Im p_{\varphi}=-2 \alpha \sum x_{i} \xi_{i} r^{-\alpha-2}+\sum \lambda_{i}\left(\xi^{2}-\alpha^{2} x_{i}^{2} r^{-2 \alpha-4}\right) .
$$


If $p_{\varphi}^{\Delta}, p_{\varphi}^{Q}$ are the conjugated operators of $\Delta, Q=\sum \lambda_{i} \partial_{i}^{2}$ respectively as in (12), we can write

$$
\begin{aligned}
\left\{\Re p_{\varphi}, \Im p_{\varphi}\right\}=\left\{\Re p_{\varphi}^{\Delta}, \Im p_{\varphi}^{\Delta}\right\}+ & \left\{\Re p_{\varphi}^{Q}, \Im p_{\varphi}^{Q}\right\} \\
& +\left\{\Re p_{\varphi}^{\Delta}, \Re p_{\varphi}^{Q}\right\}+\left\{\Im p_{\varphi}^{\Delta}, \Im p_{\varphi}^{Q}\right\} .
\end{aligned}
$$

Now, a simple calculation gives

$$
\begin{array}{r}
\left\{\Re p_{\varphi}^{\Delta}, \Im p_{\varphi}^{\Delta}\right\}=-4 \alpha \sum \xi_{j}^{2} r^{-\alpha-2}+4 \alpha(\alpha+2) r^{-\alpha-4}\left(\sum x_{j} \xi_{j}\right)^{2} \\
+4 \alpha^{3}(\alpha+1) r^{-3 \alpha-4}
\end{array}
$$

for the first term on the right hand side of (16) and

$$
\begin{aligned}
\left\{\Re p_{\varphi}^{Q}, \Im p_{\varphi}^{Q}\right\} & =-4 \alpha \sum \lambda_{j} \xi_{j}\left(\lambda_{j} \xi_{j} r^{-\alpha-2}-(\alpha+2) x_{j} r^{-\alpha-4} \sum \lambda_{k} x_{k} \xi_{k}\right) \\
& -4 \alpha^{3} r^{-\alpha-2} \sum \lambda_{j} x_{j}\left(\lambda_{j} x_{j} r^{-2 \alpha-4}-(\alpha+2) x_{j} r^{-2 \alpha-6} \sum \lambda_{k} x_{k}^{2}\right)
\end{aligned}
$$

for the second term on the right side of (16). For the third term we have

$$
\begin{aligned}
\left\{\Re p_{\varphi}^{\Delta}, \Re p_{\varphi}^{Q}\right\}=-4 \alpha^{2} \sum \xi_{j}\left(\lambda_{j} x_{j} r^{-2 \alpha-4}-\right. & \left.(\alpha+2) x_{j} r^{-2 \alpha-6} \sum \lambda_{k} x_{k}^{2}\right) \\
& -4 \alpha^{2}(\alpha+1) r^{-2 \alpha-4} \sum \lambda_{j} x_{j} \xi_{j} .
\end{aligned}
$$

Finally

$$
\begin{aligned}
\left\{\Im p_{\varphi}^{\Delta}, \Im p_{\varphi}^{Q}\right\}= & 4 \alpha^{2} r^{-\alpha-2} \sum x_{j}\left(\lambda_{j} \xi_{j} r^{-\alpha-2}-(\alpha+2) x_{j} r^{-\alpha-4} \sum \lambda_{k} x_{k} \xi_{k}\right) \\
& -4 \alpha^{2} r^{-\alpha-2} \sum \lambda_{j} x_{j}\left(\xi_{j} r^{-\alpha-2}-(\alpha+2) x_{j} r^{-\alpha-4} \sum x_{k} \xi_{k}\right),
\end{aligned}
$$

for the fourth term on the right hand side of (16).

We introduce new variables setting

$$
x=y \quad, \quad \xi=\alpha r^{-\alpha-2} \eta .
$$

We denote by $y^{2}=y \cdot y, y^{4}=(y \cdot y)^{2}$ and similarly for $\eta$. With the new notations, homogenizing in $(y, \eta)$, the pseudo-convexity condition becomes

$$
\begin{aligned}
(\alpha+1) y^{4}-\left(\sum \lambda_{j}^{2} y_{j}^{2}\right) y^{2}+(\alpha+2)\left(\sum \lambda_{j} y_{j}^{2}\right)^{2}>\left(\sum \lambda_{j}^{2} \eta_{j}^{2}\right) y^{2} \\
-(\alpha+2)\left(\sum \lambda_{j} y_{j} \eta_{j}\right)^{2}+y^{2} \eta^{2}-(\alpha+2)(y \cdot \eta)^{2} \\
+2(\alpha+2) y^{2} \sum \lambda_{j} y_{j} \eta_{j}-2(\alpha+2) y \cdot \eta \sum \lambda_{j} y_{j}^{2} .
\end{aligned}
$$

This must hold on the set $\operatorname{char} p_{\varphi}$. Taking into account (14) and (15), in $(y, \eta)$ variables this becomes

$$
\eta^{2}-y^{2}+2 \sum \lambda_{j} y_{j} \eta_{j}=0
$$

and

$$
-2 y \cdot \eta+\sum \lambda_{j}\left(\eta_{j}^{2}-y_{j}^{2}\right)=0
$$


By homogeneity we can restrict to the unit sphere $y^{2}=1$ of $\mathbf{R}_{y}^{N}$. Taking also into account (18) and (19) we can write the pseudo-convexity (17), for $y^{2}=1$, as

$$
\frac{4}{\alpha+2}<\frac{\left(1+\eta^{2}\right)^{2}+\left(\sum \lambda_{j} y_{j}^{2}+\sum \lambda_{j} \eta_{j}^{2}\right)^{2}}{1+\eta^{2}+\sum \lambda_{j}^{2} y_{j}^{2}+\sum \lambda_{j}^{2} \eta_{j}^{2}} .
$$

Hence we must study a minimization problem for the function on the right side of (20) with the constraint conditions (18), (19) and $y^{2}=1$.

By $(18), \eta$ is on a circle of radius $\sqrt{1+\sum \lambda_{j}^{2} y_{j}^{2}}$ centered at $\left(-\lambda_{j} y_{j}\right)_{1 \leq j \leq N}$.

Hence we have

$$
|\eta| \geq \sqrt{1+\sum \lambda_{j}^{2} y_{j}^{2}}-\sqrt{\sum \lambda_{j}^{2} y_{j}^{2}} .
$$

Since $y^{2}=1$ and the function $\sqrt{1+x^{2}}-x$ is decreasing on $[0,+\infty)$ it follows that

$$
|\eta| \geq \sqrt{1+\lambda^{2}}-\lambda
$$

Using (6) and (21) we obtain

$$
\begin{aligned}
\frac{\left(1+\eta^{2}\right)^{2}+\left(\sum \lambda_{j} y_{j}^{2}+\sum \lambda_{j} \eta_{j}^{2}\right)^{2}}{1+\eta^{2}+\sum \lambda_{j}^{2} y_{j}^{2}+\sum \lambda_{j}^{2} \eta_{j}^{2}} & \geq \frac{1+\eta^{2}}{1+\lambda^{2}}+\frac{\left(\sum \lambda_{j} y_{j}^{2}+\lambda_{j} \eta_{j}^{2}\right)^{2}}{\left(1+\eta^{2}\right)\left(1+\lambda^{2}\right)} \geq \\
\geq & \frac{1+\eta^{2}}{1+\lambda^{2}} \geq \frac{1+\left(\sqrt{1+\lambda^{2}}-\lambda\right)^{2}}{1+\lambda^{2}} .
\end{aligned}
$$

This bound suffices for our result. We note that it is also sharp. To see that suppose $\lambda_{1}=-\lambda$ and $\lambda_{2}=\lambda$. Then the pair

$$
y=\left(\frac{\sqrt{2}}{2}, \frac{\sqrt{2}}{2}, 0, \ldots, 0\right) \quad, \quad \eta=\left(\sqrt{1+\lambda^{2}}-\lambda\right)\left(-\frac{\sqrt{2}}{2}, \frac{\sqrt{2}}{2}, 0, \ldots, 0\right)
$$

belongs to the characteristic set of $p_{\varphi}$ and achieves equality above.

Taking into account (20) and (22), the pseudo-convexity condition holds provided that

$$
\frac{4}{\alpha+2}<\frac{1+\left(\sqrt{1+\lambda^{2}}-\lambda\right)^{2}}{1+\lambda^{2}}
$$

Using also (7) we rewrite this as

$$
\alpha>\frac{2 \lambda}{\sqrt{1+\lambda^{2}}-\lambda}=\frac{2 \sin \phi}{1-\sin \phi},
$$

which is exactly what we needed. This completes the proof of the theorem.

Proof of Theorem 2.3 - For the operator $P$ we know that we can prove Carleman estimates with the exponential weight

$$
e^{\tau \varphi}=e^{\tau r^{-\alpha}} .
$$


We first verify when we have a Carleman estimates with the exponential weight

$$
e^{\tau \varphi}=e^{\tau r^{-\alpha}}
$$

for the operator

$$
\mathcal{Q}=-\sum \nu_{k} \partial_{k}^{2}
$$

All we need is to verify the pseudo-convexity condition. The symbol of the conjugated operator is

$$
q_{\varphi}(x, \xi)=-\sum \nu_{k}\left(\xi_{k}+i \partial_{k} \varphi\right)^{2}
$$

Since

$$
\nabla \varphi=-\alpha x r^{-\alpha-2}
$$

we get

$$
\Re q_{\varphi}=\sum \nu_{k}\left(\xi_{k}^{2}-\alpha^{2} x_{k}^{2} r^{-2 \alpha-4}\right)
$$

and

$$
\Im q_{\varphi}=-2 \alpha \sum \nu_{k} x_{k} \xi_{k} r^{-\alpha-2} .
$$

Their Poisson bracket is

$$
\begin{aligned}
\left\{\Re q_{\varphi}, \Im q_{\varphi}\right\}=-2 \sum \nu_{k}^{2}\left(\xi_{k}^{2} r^{-\alpha-2}\right. & \left.+\alpha^{2} x_{k}^{2} r^{-3 \alpha-6}\right) \\
& +2 \alpha^{2}(\alpha+2) \sum \nu_{k} x_{k}^{2} \sum \nu_{j} x_{j}^{2} r^{-3 \alpha-8} .
\end{aligned}
$$

The pseudo-convexity condition reads:

$$
\left\{\Re q_{\varphi}, \Im q_{\varphi}\right\}>0 \quad \text { on } \quad \Re q_{\varphi}=\Im q_{\varphi}=0 .
$$

With the new notations

$$
\xi_{k}=\nu_{k}^{-\frac{1}{2}} \alpha r^{-\alpha-2} \eta_{k} \quad, \quad x_{k}=\nu_{k}^{-\frac{1}{2}} y_{k}
$$

the pseudo-convexity condition becomes

$$
(\alpha+2) y^{4}>\left(\sum \nu_{k}\left(y_{k}^{2}+\eta_{k}^{2}\right)\right)\left(\sum \nu_{k}^{-1} y_{k}^{2}\right) \quad \text { on } \quad y^{2}=\eta^{2} ; y \cdot \eta=0 .
$$

Fix $y^{2}=\eta^{2}=1$ and assume that

$$
0<\nu_{1} \leq \ldots \leq \nu_{N}
$$

Maximize the right hand side of (24) with respect to $\eta$. Then we need 


$$
(\alpha+2)>E=\left(\nu_{N}+\sum_{1}^{N-1} \nu_{k} y_{k}^{2}\right)\left(\sum_{1}^{N-1} \nu_{k}^{-1} y_{k}^{2}\right), \quad y^{2}=1 .
$$

Maximizing the right hand side with respect to $\eta$, this becomes

$$
\alpha+2>\max _{y^{2}=\eta^{2}=1 ; y \cdot \eta=0} E=\left(\nu_{N}+\nu_{1}\right) \nu_{1}^{-1}=1+\frac{\nu_{N}}{\nu_{1}} .
$$

Thus the pseudo-convexity condition holds provided that

$$
\alpha>\frac{\nu_{N}-\nu_{1}}{\nu_{1}} \text {. }
$$

We now return to our problem and make the following change of variables

$$
\left\{\begin{array}{l}
X_{1}=\sqrt{\mu_{1}} x_{1} \\
\vdots \\
X_{N}=\sqrt{\mu_{N}} x_{N}
\end{array}\right.
$$

The operators $\Delta$ and $\mu_{1}^{2} \partial_{x_{1}}^{2}+\cdots+\mu_{N}^{2} \partial_{x_{N}}^{2}$ become, respectively,

$$
\frac{1}{\mu_{1}} \partial_{X_{1}}^{2}+\cdots+\frac{1}{\mu_{N}} \partial_{X_{N}}^{2} \quad \text { and } \quad \mu_{1} \partial_{X_{1}}^{2}+\cdots+\mu_{N} \partial_{X_{N}}^{2}
$$

By (25), the weight function $\varphi$ satisfies the pseudo-convexity condition with respect to both transformed operators if we take

$$
\alpha>\frac{\max _{i, j}\left|\mu_{i}-\mu_{j}\right|}{\min _{i} \mu_{i}} .
$$

This transfers to the operators $P$ and $Q$ since without any restriction in generality we can freeze their coefficients at 0 , just as in the proof of Theorem 2.2.

Thus we have Carleman estimates for both $P$ and $Q$ with the weight $\varphi$.

Putting these two together we easily obtain a Carleman estimate for the product. Then we can conclude in the standard way.

\section{Appendix}

We begin with two lemmas which we have used (see [16]).

Lemma 3.1. Let be $\nu>0$ and $r^{2}(x)$ a positive quadratic form in $\mathbf{R}^{N}$; then the function $u(x)=\exp \left(-r^{-\nu}\right)$ belongs to $G^{1+\nu^{-1}}\left(\mathbf{R}^{N}\right)$.

Lemma 3.2. Let $\Omega$ be an open neighborhood of the origin in $\mathbf{R}^{N}$ and $u \in G^{s}(\Omega)$. If $u$ is flat at zero, then there exists a function $v \in C^{\infty}(\Omega)$ flat at zero such that

$$
u=\exp \left(-r^{-\nu}\right) v
$$

provided $1+\nu^{-1}>s$. 
We state now the principal results in [16].

Theorem 3.3. ([16], pag. 1165) Let $P$ be a second order, elliptic operator with Gevrey coefficients of order $s>1$, defined in an open neighborhood of the origin in $\mathbf{R}^{2}$ and $p$ its principal symbol.

1. If $p\left(0, \mathbf{R}^{2} \backslash\{0\}\right)$ is a convex cone with angle $2 \phi^{1}, 0<\phi<\frac{\pi}{2}$ and if

$$
s<1+\frac{1-\sin \phi}{2 \sin \phi}
$$

then $P$ has the strong unique continuation property at zero.

2. If $p\left(0, \mathbf{R}^{2} \backslash\{0\}\right)=\mathbf{C} \backslash\{0\}$ and if $P$ has simple characteristic, then there exists a real number $\sigma_{0}>1$ depending only on $P\left(0, D_{x}\right)$ such that $P$ has the strong unique continuation property at zero for any $s<\sigma_{0}$.

In $\mathbf{R}^{N}(N>2)$ we have

Theorem 3.4. ([16], pag. 1165) Under the hypothesis of the foregoing theorem, there exists $\sigma_{0}>1$, depending only on $p\left(0, D_{x}\right)$ such that $P$ has the strong unique continuation property at zero for any $s<\sigma_{0}$.

The idea of the proof in Theorem 3.3 is that if $P$ is a second order, elliptic operator with smooth coefficients defined in an open neighborhood of the origin in $\mathbf{R}^{2}$ and if it has simple characteristics then there exist two smooth, elliptic vector fields $X_{1}, X_{2}$ such that

$$
P=X_{1} X_{2}+P_{1}
$$

where $P_{1}$ is a first order differential operator with coefficients $C^{\infty}$.

Now, if $X$ is a smooth, elliptic vector field then we set

$$
X_{\gamma}=e^{-\gamma \varphi} X e^{\gamma \varphi}
$$

where $\varphi=-\nu^{-1} r^{-\nu}(\nu>0)$ and $r=|x|$. If we choose $\nu$ such that

$$
\nu+2>\frac{|X(0)|^{2}}{\min _{|x|=1}|\langle X(0), x\rangle|^{2}},
$$

we can give a Carleman estimate for $X$. Then if we iterate Carleman estimates for $X_{1}, X_{2}$ fields in (28) we obtain a Carleman estimate for $P$ by using $P_{\gamma}=$ $e^{-\gamma \varphi} P e^{\gamma \varphi}$.

\footnotetext{
${ }^{1}$ in this case, it is easy to see that $P$ has simple characteristics
} 
When $p$ verifies the cone property of angle $2 \phi, 0 \leq \phi<\frac{\pi}{2}$, we can find coordinates such that the relation (29) can be written, for any vector field, as

$$
\nu>\frac{2 \sin \phi}{1-\sin \phi} .
$$

Remark 3.1. We note that if $\nu$ is as in (30) from (13) it follows that the strong unique continuation property holds again for functions $u$ such that:

1. $u=e^{\gamma \varphi} v_{\gamma}$ for any $\gamma \gg 0$ and $v_{\gamma} \in C^{\infty}$ flat at zero and $\varphi$ as above.

2. $|P u(x)| \leq \epsilon\left(\frac{|u(x)|}{|x|^{\frac{3}{2} \nu+2}}+\frac{|\nabla u(x)|}{|x|^{\frac{\nu}{2}+1}}\right), x \in \Omega$ neighborhood of zero, for some constant $\epsilon>0$ small enough.

This strong uniqueness result holds also if the condition (1) in Remark 3.1 is replaced with a weaker one as

$$
\int_{|x| \leq R}|u(x)|^{2} d x=\mathcal{O}\left(e^{-R^{-\nu}}\right)
$$

when $R \rightarrow 0$.

Similar remarks hold for Theorem 2.3.

\section{References}

[1] S. Alinhac, Non-unicité pour des opérateurs différentiels à caractéristiques complexes simples, Ann. Sci. École Norm. Sup. 13 (1980) 385-393.

[2] S. Alinhac and M.S.Baouendi, Uniqueness for the characteristic Cauchy problem and strong unique continuation for higher order partial differential inequalities, Amer. J. Math. 102 (1980) 179-217.

[3] _ A counterexample to strong uniqueness for partial differential equations of Schrödinger's type, Comm. Partial Differential Equations 19 (1994) 1727-1733.

[4] S. Alinhac and N. Lerner, Unicité forte à partir d'une variété de dimension quelconque pour des inégalités différentielles elliptiques, Duke Math. J. 48 (1981) 49-68.

[5] F. Colombini and C. Grammatico, Some remarks on strong unique continuation for the Laplace operator and its powers, Comm. Partial Differential Equations 24 (1999) 10791094.

[6] _ Strong uniqueness in Gevrey spaces for some elliptic operators, in Hyperbolic differential operators and related problems (V.Ancona and J.Vaillant eds.), Marcel Dekker, New York Basel 2003, 301-316.

[7] _ _ A counterexample to strong uniqueness for all powers of the Laplace operator, Comm. Partial Differential Equations 25 (2000) 585-600.

[8] _ A result on strong uniqueness in Gevrey spaces for some elliptic operators, Comm. Partial Differential Equations 30 (2005) 39-57.

[9] C. Grammatico, A result on strong unique continuation for the Laplace operator, Comm. Partial Differential Equations 22 (1997) 1475-1491.

[10] L. Hörmander, Linear partial differential operators, Springer Verlag, Berlin, 1963.

[11] _ Uniqueness theorems for second order elliptic differential equations, Comm. Partial Differential Equations 8 (1983) 21-64.

[12] D. Jerison and C. E. Kenig, Unique continuation and absence of positive eigenvalues for Schrödinger operator, Ann. of Math. 121 (1985) 463-494. 
[13] H. Koch and D. Tataru, Sharp counterexamples in unique continuation for second order elliptic equations, J. Reine Angew. Math. 542 (2002) 133-146.

[14] _ Recent results on unique continuation for second order elliptic equations, in Carleman estimates and applications to uniqueness and control theory (F.Colombini and C.Zuily eds.), Progr. Nonlinear Differential Equations Appl. 46, Birkhäuser, Boston, 2001, 73-84.

[15] _ Carleman estimates and unique continuation for second order elliptic equations with nonsmooth coefficients, Comm. Pure Appl. Math. 54 (2001) 339-360.

[16] N. Lerner, Résultats d'unicité forte pour des opérateurs elliptiques à coefficients Gevrey, Comm. Partial Differential Equations 6 (1981) 1163-1177.

[17] N. Mandache, A counterexample to unique continuation in dimension two, Comm. Anal. Geom. 10 (2002) 1435-1444.

[18] Y. Pan, Unique continuation for Schrödinger operators with singular potentials, Comm. Partial Differential Equations 17 (1992) 953-965.

[19] R. Regbaoui, Strong unique continuation for second order elliptic differential operators, J. Differential Equations 141 (1997) 201-217.

[20] J. Sjöstrand, Parametrices for pseudo-differential operators with multiple characteristics, Ark. Mat. 12 (1974) 85-130.

[21] C. D. Sogge, Oscillatory integrals and unique continuation for second order elliptic differential equations, J. Amer. Math. Soc. 2 (1989), no. 3, 491-515.

[22] D. Tataru, Carleman estimates, unique continuation and applications, http://math.berkeley.edu/ tataru/ucp.html

[23] T.Wolff, A counterexample in a unique continuation problem, Comm. Anal. Geom. 2 (1994) 79-102.

Dipartimento di Matematica, Università di Pisa, Largo B.Pontecorvo 5, 56127

Pisa, Italy

E-mail address: colombini@dm.unipi.it

Dipartimento di Matematica \& CiRAM, Università di Bologna, via Saragozza 8, 40123 BolognA, ITALY

E-mail address: grammati@dm.unibo.it

Department of Mathematics, University of California, Berkeley, Berkeley, CA 94720

E-mail address: tataru@math.berkeley.edu 